\title{
A Reflection of a Taiwanese Resident of Surgery in COVID-19 Pandemic
}

\author{
Ting-Wei Chang ${ }^{1} \cdot$ Po-Chih Chang ${ }^{1,2}(\mathbb{D}$
}

Received: 31 January 2022 / Revised: 2 February 2022 / Accepted: 3 February 2022 / Published online: 14 February 2022

(c) The Author(s), under exclusive licence to Springer Science+Business Media, LLC, part of Springer Nature 2022

\begin{abstract}
In March 2020, the coronavirus disease 2019 (COVID-19) outbreak was declared as a pandemic by the World Health Organization. The COVID-19 breached the Taiwanese government's defense, and the Central Epidemic Command Center (CECC) announced a Nationwide Level 3 Alert on May 19, 2021. In summary, the residency training has been affected as follows: less exposure to elective surgeries and decreased training time. In the COVID-19 pandemic era, both my life and career as a surgical resident have been deeply affected. Worries, anxiety, and fear about insufficient training, unfulfilled career development, and unknown future have been prevalent daily. However, surgeons have always managed to confront and overcome difficulties and challenges with courage and fearlessness. Hence, it is also important that we face the COVID-19 pandemic situation and adapt wisely.
\end{abstract}

Keywords COVID-19 $\cdot$ Resident $\cdot$ Surgery

In March 2020, the coronavirus disease 2019 (COVID19) outbreak was declared as a pandemic by the World Health Organization (WHO). The COVID-19 pandemic started in China and rapidly spread worldwide. Taiwan, a non-WHO member and an important transportation hub located nearby China, was also affected in various aspects during this pandemic. The Ministry of Health and Welfare of Taiwan responded rapidly to the COVID-19 outbreak, including enhanced border control and quarantine measures,

Key Points The COVID-19 breached Taiwanese government defense, and a Nationwide Level 3 Alert was announced on May 19, 2021.

After the Nationwide Level 3 Alert, compartmentalization and alternate working days were introduced at my training hospital to prevent cross infections.

During the COVID-19 pandemic, the residents in the tertiary medical center support the community vaccination stations and the COVID-19 community screening stations.

The surgical residency training has been affected as follows: less exposure to elective surgeries and decreased training time.

Po-Chih Chang

dyno910076@hotmail.com

1 Division of Thoracic Surgery, Department of Surgery, Kaohsiung Medical University Hospital, Kaohsiung Medical University, Kaohsiung City, Taiwan

2 Weight Management Center, Kaohsiung Medical University Hospital, Kaohsiung Medical University, No. 100, Tzyou 1st Road, Kaohsiung City 80756, Taiwan establishment of the Central Epidemic Command Center (CECC) for developing domestic infection control guidelines and for the coordination between the government department and private stakeholders, and the application of information technology combined with the national health insurance [1, 2]. However, COVID-19 still breached our defense, and the CECC announced a Nationwide Level 3 Alert on May 19, 2021. Since then, my surgical residency training program and bariatric surgery training plan have become different.

I am a surgical resident in a tertiary medical center in Taiwan, and this is the third year of my 5-year residency program, which was influenced by the COVID-19 pandemic for approximately two-thirds of my current residency. In summary, the residency training has been affected as follows: less exposure to elective surgeries and decreased training time.

\section{Less Exposure to Elective Surgeries}

During the Nationwide Level 3 Alert, my training hospital postponed most elective surgeries, including bariatric surgeries, until the end of the Nationwide Level 3 Alert. The volume of elective surgeries was reduced to half compared to the same period last year, and bariatric surgeries were all postponed until the end of the Nationwide Level 3 Alert. 


\section{Decreased Training Time}

After the Nationwide Level 3 Alert, compartmentalization and alternate working days were introduced at my training hospital to prevent cross infections. Junior residents of surgery (first- and second-year residency) stayed on the floor of the general ward and were divided into two groups. Orderly, each group took turns coming to the hospital for work for 2 weeks and then resting at home for 2 weeks. Therefore, the junior residents were unable to join the surgery in the operation theater during the Nationwide Level 3 Alert. The senior residents of surgery (third- to fifth-year residency) were also divided into two groups: one group stayed in the intensive care units and the other group worked in the operating room. Individuals switched groups every 2 weeks. Nowadays, an obvious effect of compartmentalization and alternate working days has been one of the milestones for junior residents: totally implantable venous access device implantation. Consequently, junior residents have less confidence and familiarity compared to junior residents who had not experienced the COVID-19 pandemic.

During the COVID-19 pandemic, the residents in the tertiary medical center support the community vaccination stations and the COVID-19 community screening stations. Taiwan CECC established huge community vaccination stations to increase the coverage of COVID-19 vaccination, and community screening stations for patients being admitted and citizens traveling back from overseas. On average, I have a half working day each week to support the community vaccination stations or screening stations, which may coincide with the day of my surgical attendance, thus compromising my ability to participate and obtain clinical practice experience.

\section{Modification of My Residency Training}

Certain modifications have been made to overcome this situation. Each training module is posted online in a step-by-step video of laparoscopic and thoracoscopic surgery, so that we could review and gain familiarity with these surgical procedures. We have also increased the time of clinical practice via the laparoscopic training box to allow participants to be educated on manipulating instruments and on learning the intracorporeal suture technique, which enhanced greatly the staple line reinforcement in laparoscopic sleeve gastrectomy. I also participated in the fifth international forum of International Excellence Federation For Bariatric and Metabolic
Surgery, held by Dr. Huang, Chih-Kun. Due to the less time spent in the operating room, I have instead spent more time in the study room. Moreover, I have started organizing the cases and data of my attendings and my department, and I have published them as a scientific documentation. For instance, I have described my revision surgery experience regarding gastric clip treatment in patients with morbid obesity in detail, and our perspectives on different sizes of boogie calibration for laparoscopic sleeve gastrectomy [3, 4].

In the COVID-19 pandemic era, both my life and career as a surgical resident have been deeply affected. Worries, anxiety, and fear about insufficient training, unfulfilled career development, and unknown future have been prevalent daily. However, surgeons have always managed to confront and overcome difficulties and challenges with courage and fearlessness. Hence, it is also important that we face the COVID-19 pandemic situation and adapt wisely.

\section{Declarations}

Conflict of Interest The authors declare no competing interests.

Ethical Approval This article does not contain any studies with human participants or animals performed by any of the authors.

Informed Consent Informed consent does not apply.

\section{References}

1. Su SF, Han YY. How Taiwan, a non-WHO member, takes actions in response to COVID-19. J Glob Health. 2020;10(1):010380. https://doi.org/10.7189/jogh.10.010380.

2. Chen C-C, Tseng C-Y, Choi W-M, et al. Taiwan governmentguided strategies contributed to combating and controlling COVID-19 pandemic. Front Public Health. 2020;8:547423. https://doi.org/10.3389/fpubh.2020.547423.

3. Chang, PC., Chen, KH., Jhou, HJ. et al. Promising effects of 33 to $36 \mathrm{Fr}$. bougie calibration for laparoscopic sleeve gastrectomy: a systematic review and network meta-analysis. Sci Rep 11, 15217 (2021). https://doi.org/10.1038/s41598-021-94716-1

4. Chang PC, Chen KH, Huang IY, et al. Laparoscopic revision for gastric clipping: a single center experience and Taiwan database review. Obes Surg. 2021;31(8):3653-9. https://doi.org/10.1007/ s11695-021-05466-y.

Publisher's Note Springer Nature remains neutral with regard to jurisdictional claims in published maps and institutional affiliations. 